\title{
Intrabeam scattering in electron storage rings
}

\author{
Kiyoshi Kubo and Katsunobu Oide \\ KEK, High Energy Accelerator Research Organization, 1-1 Oho, Tsukuba, Ibaraki 305-0801, Japan
}

(Received 6 September 2001; published 7 December 2001)

A calculation method of emittance growth of an electron beam due to intrabeam scattering is described. The 3 degrees of freedom are equally treated in the beam rest frame, and the couplings between them are included in a natural way. This formalism is suitable for the calculation of the emittance growth with the beam-envelope method.

DOI: 10.1103/PhysRevSTAB.4.124401

PACS numbers: 29.27.Fh, 29.20.Dh, 41.75.Ht

\section{INTRODUCTION}

In the past few years, the emittance growth due to the intrabeam scattering has been an important issue in electron storage rings for electron-positron linear colliders and synchrotron light sources. Even though the basic theoretical framework was established by Piwinski [1] many years ago and developed by other authors [2,3], a precise comparison between the theory and the experiment was made possible only recently [4-6]. In this paper we intend to clarify the basic formula again and restate its essence to be applicable to a general situation with couplings between the degrees of freedom. This formalism is suitable for the numerical calculation of the emittance growth with the beamenvelope method and used in a computer program SAD [7] for calculating the equilibrium beam emittance in an electron storage ring.

\section{BJORKEN AND MTINGWA (BM) FORMULA}

We start from the formula of Bjorken and Mtingwa (BM) [2] because it is ready for generalization for coupled beams. Only scattering of two particles close to each other is taken into account, or scattering is assumed to take place locally in the space. In our paper, everything is described in the beam rest frame unless explicitly noted. Note also that our notations are not the same as BM.
Let us consider a bunched beam and assume that the phase-space distribution of the beam is Gaussian. Following BM, $\rho(x, p)$, the electron's phase space density, can be expressed as

$$
\rho(x, p)=\frac{N}{\Gamma} e^{-S(x, p)},
$$

with

$$
\begin{aligned}
S(x, p)=\sum_{i, j=1}^{3}( & \frac{1}{2} A_{i j} \delta p_{i} \delta p_{j} \\
& \left.+B_{i j} \delta p_{i} \delta x_{j}+\frac{1}{2} C_{i j} \delta x_{i} \delta x_{j}\right),
\end{aligned}
$$

and

$$
\Gamma=\int d^{3} x d^{3} p e^{-S(x, p)},
$$

where $\delta \vec{p}$ and $\delta \vec{x}$ are the momentum and coordinate deviation from the average and $N$ is the number of electrons in the beam.

A rate of electron-electron scattering, in which the 4-momentum of two particles changes from $\left(p_{1}, p_{2}\right)$ to $\left(p_{1}^{\prime}, p_{2}^{\prime}\right)$, is expressed as [8]

$$
\begin{gathered}
\frac{d N}{d t}=\frac{1}{2} \int d^{3} x \rho\left(x, p_{1}\right) \rho\left(x, p_{2}\right) \int \frac{m d^{3} p_{1}^{\prime}}{(2 \pi)^{2} E_{1}^{\prime}} \int \frac{m d^{3} p_{2}^{\prime}}{(2 \pi)^{2} E_{2}^{\prime}} \frac{m^{2}}{E_{1} E_{2}}|M|^{2}(2 \pi)^{4} \delta^{4}\left(p_{1}^{\prime}+p_{2}^{\prime}-p_{1}-p_{2}\right), \\
|M|^{2}=\frac{(4 \pi \alpha)^{2}}{2 m^{4}}\left\{\frac{\left(p_{1} \cdot p_{2}\right)^{2}+\left(p_{1} \cdot p_{2}^{\prime}\right)^{2}+2 m^{2}\left(p_{1} \cdot p_{2}^{\prime}-p_{1} \cdot p_{2}\right)}{\left[\left(p_{1}^{\prime}-p_{1}\right)^{2}\right]^{2}}\right. \\
\left.+\frac{\left(p_{1} \cdot p_{2}\right)^{2}+\left(p_{1} \cdot p_{1}^{\prime}\right)^{2}+2 m^{2}\left(p_{1} \cdot p_{1}^{\prime}-p_{1} \cdot p_{2}\right)}{\left[\left(p_{2}^{\prime}-p_{1}\right)^{2}\right]^{2}}+2 \frac{\left(p_{1} \cdot p_{2}\right)^{2}-2 m^{2} p_{1} \cdot p_{2}}{\left(p_{1}^{\prime}-p_{1}\right)^{2}\left(p_{2}^{\prime}-p_{1}\right)^{2}}\right\},
\end{gathered}
$$

where $E_{1}, E_{2}, E_{1}^{\prime}$, and $E_{2}^{\prime}$ are the electron energies corresponding to their momentum, and $m$ is the electron mass.

For a nonrelativistic scattering [9],

$$
|M|^{2} \approx(4 \pi \alpha)^{2}\left\{\frac{1}{q^{4}}-\frac{3}{4 q^{2}\left(p_{1}-p_{2}\right)^{2}}\right\},
$$

where $q=p_{1}^{\prime}-p_{1}$. In the center-of-mass frame of the two particles, 


$$
q^{2}=-2\left|\vec{p}_{1}\right|^{2}(1-\cos \theta)
$$

and

$$
\left(p_{1}-p_{2}\right)^{2}=-4\left|\vec{p}_{1}\right|^{2},
$$

where $\theta$ is the scattering angle. Because scattering with small angle is dominant, from now on the approximation as follows will be used:

$$
|M|^{2} \approx(4 \pi \alpha)^{2} \frac{1}{q^{4}} .
$$

Let us consider the average of the second order product of momentum, $\left\langle p_{i} p_{j}\right\rangle,(i, j=1,2,3)$. Here the angle bracket indicates the average over all particles in a bunch. From the equations above, change of this average in a short period of time $\Delta t$ can be expressed as follows:

$$
\begin{aligned}
\frac{\Delta\left\langle p_{i} p_{j}\right\rangle}{\Delta t}= & \frac{1}{2} \int d^{3} x \rho\left(x, p_{1}\right) \rho\left(x, p_{2}\right) \int \frac{m d^{3} p_{1}^{\prime}}{(2 \pi)^{2} E_{1}^{\prime}} \int \frac{m d^{3} p_{2}^{\prime}}{(2 \pi)^{2} E_{2}^{\prime}} \frac{m^{2}}{E_{1} E_{2}}|M|^{2}(2 \pi)^{4} \delta^{4}\left(p_{1}^{\prime}+p_{2}^{\prime}-p_{1}-p_{2}\right) \\
& \times\left\{\left(p_{1}^{\prime}\right)_{i}\left(p_{1}^{\prime}\right)_{j}-\left(p_{1}\right)_{i}\left(p_{1}\right)_{j}+\left(p_{2}^{\prime}\right)_{i}\left(p_{2}^{\prime}\right)_{j}-\left(p_{2}\right)_{i}\left(p_{2}\right)_{j}\right\}
\end{aligned}
$$

Using the average of the momentum in the beam, $\langle\vec{p}\rangle$, and introducing $\vec{\xi}$ and $\vec{\Delta}$ as

$$
\begin{gathered}
\vec{\xi} \equiv \vec{p}_{1}+\vec{p}_{2}-2\langle\vec{p}\rangle, \\
\vec{\Delta} \equiv \frac{\vec{p}_{1}-\vec{p}_{2}}{2}, \\
\int d^{3} p_{1} \int d^{3} p_{1}=\int d^{3} \xi \int d \Delta^{3},
\end{gathered}
$$

the last factor of Eq. (8) becomes [10]

$$
\begin{gathered}
\left(p_{1}^{\prime}\right)_{i}\left(p_{1}^{\prime}\right)_{j}-\left(p_{1}\right)_{i}\left(p_{1}\right)_{j}+\left(p_{2}^{\prime}\right)_{i}\left(p_{2}^{\prime}\right)_{j}-\left(p_{2}\right)_{i}\left(p_{2}\right)_{j} \\
=2\left(q_{i} \Delta_{j}+q_{j} \Delta_{i}+q_{i} q_{j}\right)
\end{gathered}
$$

From Eqs. (1) and (2),

$$
\rho\left(x, p_{1}\right) \rho\left(x, p_{2}\right)=\frac{N^{2}}{\Gamma^{2}} e^{-\left[S\left(x, p_{1}\right)+S\left(x, p_{2}\right)\right]},
$$

and

$$
S\left(x, p_{1}\right)+S\left(x, p_{2}\right)=S\left(\sqrt{2} x, \frac{\xi}{\sqrt{2}}\right)+\tilde{S},
$$

with

$$
\tilde{S} \equiv \sum_{i, j=1}^{3} A_{i j} \Delta_{i} \Delta_{j} .
$$

Using nonrelativistic approximation,

$$
E_{1} \approx E_{2} \approx E_{1}^{\prime} \approx E_{2}^{\prime} \approx m,
$$

the integration of $x$ and $\xi$ can be done straightforwardly, and Eq. (10) becomes

$$
\frac{\Delta\left\langle p_{i} p_{j}\right\rangle}{\Delta t}=\frac{4 \alpha^{2} N}{\Gamma} \int d^{3} \Delta e^{-\tilde{S}}\left(I_{i} \Delta_{j}+I_{j} \Delta_{i}+J_{i j}\right),
$$

where $I_{i}$ and $J_{i j}$ are the spatial components of the 4-vector, $I_{\mu}$, and the 4-tensor, $J_{\mu \nu}$,

$$
I_{\mu} \equiv \int \frac{d^{3} p_{1}^{\prime}}{(2 \pi)^{2}} \int \frac{d^{3} p_{2}^{\prime}}{(2 \pi)^{2}} \frac{q_{\mu}}{q^{4}} \delta^{4}\left(p_{1}^{\prime}+p_{2}^{\prime}-p_{1}-p_{2}\right),
$$

$$
\begin{aligned}
J_{\mu \nu} \equiv & \int \frac{d^{3} p_{1}^{\prime}}{(2 \pi)^{2}} \int \frac{d^{3} p_{2}^{\prime}}{(2 \pi)^{2}} \frac{q_{\mu} q_{\nu}}{q^{4}} \\
& \times \delta^{4}\left(p_{1}^{\prime}+p_{2}^{\prime}-p_{1}-p_{2}\right) .
\end{aligned}
$$

Note that indices $i, j$, and $k$ go from 1 to 3 , whereas $\mu$ and $\nu$ go from 0 to 3 .

Let us first consider the center-of-mass system (CMS) of the two scattering particles because the integrations can be performed easily in this coordinate system. We choose the direction of the CMS as

$$
\begin{gathered}
\vec{\Delta}=(0,0,|\vec{\Delta}|), \\
q_{0}=0, \\
\vec{q}=|\vec{\Delta}|(\sin \theta \cos \phi, \sin \theta \sin \phi, 1-\cos \theta),
\end{gathered}
$$

where $\theta$ and $\phi$ are the scattering angles. The results of the integrations are as follow:

$$
\begin{gathered}
I_{0}=I_{1}=I_{2}=0, \\
I_{3}=-\frac{\pi m}{4|\vec{\Delta}|^{2}} \int d(\cos \theta) \frac{1}{1-\cos \theta}, \\
J_{\mu \nu}=0, \quad(\mu \neq \nu), \\
J_{00}=0, \\
J_{11}=J_{22}=\frac{\pi m}{8|\vec{\Delta}|} \int d(\cos \theta) \frac{1+\cos \theta}{1-\cos \theta},
\end{gathered}
$$

and

$$
J_{33}=\frac{\pi m}{4|\vec{\Delta}|} \int d(\cos \theta) .
$$

The integrals in Eqs. (26) and (29) are divergent for small scattering angles. Here, let us simply denote this integral as (log), so called "Coulomb logarithm," 


$$
\begin{aligned}
(\log ) & =\frac{1}{2} \int d(\cos \theta) \frac{1}{1-\cos \theta} \\
& \approx \frac{1}{4} \int d(\cos \theta) \frac{1+\cos \theta}{1-\cos \theta} \\
& \gg \int d(\cos \theta),
\end{aligned}
$$

and discuss it later.

Now, let us express the integrations in a general coordinate system. As can be seen from Eqs. (20) and (21), $I_{\mu}$ and $J_{\mu \nu}$ depend only on the momentum of the two scattering particles, $p_{1}$ and $p_{2}$, and can be expressed as a function of 4 -vectors $W$ and $\Delta$ which are defined as

$$
\begin{aligned}
& W_{\mu}=\left(p_{1}\right)_{\mu}+\left(p_{2}\right)_{\mu}, \\
& \Delta_{\mu}=\frac{\left(p_{1}\right)_{\mu}-\left(p_{2}\right)_{\mu}}{2} .
\end{aligned}
$$

From Eqs. (25)-(30), and considering that $I_{\mu}$ is a Lorentz vector and $J_{\mu \nu}$ is a Lorentz tensor, these can be expressed in a general coordinate system as

$$
I_{\mu}=\frac{\pi m}{2\left|\Delta^{2}\right|^{3 / 2}}(\log ) \Delta_{\mu}
$$

and

$J_{\mu \nu}=-\frac{\pi m}{2 \sqrt{\Delta^{2}}}(\log )\left(g_{\mu \nu}-\frac{W_{\mu} W_{\nu}}{W^{2}}-\frac{\Delta_{\mu} \Delta_{\nu}}{\left|\Delta^{2}\right|}\right)$,

where

$$
\begin{aligned}
g_{\mu \nu}=g^{\mu \nu} & =\left(\begin{array}{cccc}
1 & 0 & 0 & 0 \\
0 & -1 & 0 & 0 \\
0 & 0 & -1 & 0 \\
0 & 0 & 0 & -1
\end{array}\right) \\
\Delta^{2} & \equiv \sum_{\mu, \nu=0}^{3} g^{\mu \nu} \Delta_{\mu} \Delta_{\nu}
\end{aligned}
$$

and

$$
W^{2} \equiv \sum_{\mu, \nu=0}^{3} g^{\mu \nu} W_{\mu} W_{\nu} .
$$

From these results, Eq. (19) becomes

$$
\begin{aligned}
\frac{\Delta\left\langle p_{i} p_{j}\right\rangle}{\Delta t}= & \frac{2 \pi \alpha^{2} m N}{\Gamma}(\log ) \\
& \times \int \frac{d^{3} \Delta}{|\vec{\Delta}|} e^{-\tilde{S}}\left(\delta_{i j}-3 \frac{\Delta_{i} \Delta_{j}}{|\vec{\Delta}|^{2}}\right) .
\end{aligned}
$$

Here the calculation is in the rest frame of the beam, we assumed that the particles are nonrelativistic in the frame, then we ignored the term $W_{i} W_{j} / W^{2}$,

$$
\frac{W_{i} W_{j}}{W^{2}} \sim\left(\frac{v}{c}\right)^{2} \ll 1, \quad(i, j=1,2,3),
$$

where $v$ is the typical particle velocity in the beam rest frame.

Considering the definition of $\vec{\Delta}$, Eq. (12), and its distribution, $\vec{\Delta}$ can be replaced by $\delta \vec{p} / \sqrt{2}$. Here, $\delta \vec{p}$ denotes deviation of $\vec{p}$ from the local average, where we should average over particles at the same position, $\vec{x}_{1}=\vec{x}_{2}$, because scattering is assumed to take place locally,

$$
\vec{\Delta} \rightarrow \frac{\delta \vec{p}}{\sqrt{2}} .
$$

Let us introduce a vector

$$
\vec{\omega} \equiv 2 \vec{\Delta} \rightarrow \sqrt{2} \delta \vec{p} .
$$

Then, $\tilde{S}$ can be expressed as

$$
\tilde{S}=\frac{1}{4} \vec{\omega}^{T} A \vec{\omega},
$$

where $A$ is the $3 \times 3$ matrix in Eqs. (2) and (17).

Finally, we obtain

$$
\begin{aligned}
\frac{\Delta\left\langle\delta p_{i} \delta p_{j}\right\rangle}{\Delta t}= & \frac{\pi \alpha^{2} m N(\log )}{2 \Gamma} \\
& \times \sum_{i, j=1}^{3} \int \frac{d^{3} \omega e^{-\tilde{S}}}{|\omega|^{3}}\left(\delta_{i j}|\omega|^{2}-3 \omega_{i} \omega_{j}\right),
\end{aligned}
$$

which gives the momentum diffusion rate in the beam rest frame. Basically the same equation is in BM, where the rate is expressed in the laboratory frame. The rate expressed in the beam rest frame is different from the rate expressed in the laboratory frame by the energy factor $\gamma$ of the beam. This is due to a relativistic time expansion

$$
\Delta t=\frac{\Delta t_{\mathrm{lab}}}{\gamma}
$$

where $\Delta t_{\text {lab }}$ is the time in the laboratory frame which corresponds to the $\Delta t$ in the beam rest frame.

Considering Eqs. (1) and (2), the $3 \times 3$ matrix $A$ is expressed as

$$
A=F^{-1}
$$

where $F$ is the local average of momentum matrix,

$$
F_{i j} \equiv\left\langle\delta p_{i} \delta p_{j}\right\rangle .
$$

In BM, approximations are used, such as assuming no horizontal-vertical and vertical-longitudinal coupling. Then, using beam parameters in the laboratory frame, $A$ is expressed as 


$$
\begin{aligned}
A \approx & \frac{1}{p_{\text {beam }}^{2}} \\
& \times\left(\begin{array}{ccc}
\frac{\beta_{x}}{\epsilon_{x}} & 0 & -\frac{\beta_{x}}{\epsilon_{x}} \gamma \phi \\
0 & -\frac{\beta_{y}}{\epsilon_{y}} & 0 \\
-\frac{\beta_{x}}{\epsilon_{x}} \gamma \phi & 0 & \frac{\gamma^{2}}{\sigma_{\delta}^{2}}+\frac{\beta_{x}}{\epsilon_{x}}\left(\frac{\gamma^{2} \eta_{x}^{2}}{\beta_{x}^{2}}+\gamma^{2} \phi^{2}\right)
\end{array}\right),
\end{aligned}
$$

where $p_{\text {beam }}$ is the average beam momentum, $\epsilon_{x}$ the horizontal emittance, $\epsilon_{y}$ the vertical emittance, $\eta_{x}$ the horizontal dispersion, $\sigma_{\delta}$ the relative energy spread [11], and

$$
\phi=\eta_{x}^{\prime}+\frac{\alpha_{x} \eta_{x}}{\beta_{x}}
$$

Such approximations are not necessary, and we propose to treat all three axes equally and include couplings in a general way. Diagonalizing the $3 \times 3$ matrices $F$ and $A$ makes it possible, as we explain in the following.

\section{DIAGONALIZATION}

First, let us consider diagonalization of matrix $A$ to simplify the integration in Eq. (44). Since the local average of $3 \times 3$ momentum matrix $F$ is symmetric and positive definite, it can be diagonalized as

$$
G=R F R^{T}=\left(\begin{array}{ccc}
u_{1} & 0 & 0 \\
0 & u_{2} & 0 \\
0 & 0 & u_{3}
\end{array}\right)
$$

by an orthogonal matrix, $R$, which satisfies

$$
R^{T}=R^{-1} \text {. }
$$

Let us define "normal momentum" $\delta \vec{w}$ as

$$
\delta \vec{w} \equiv R \delta \vec{p}
$$

then the matrix $G$ represents average of second order of momentum as follows:

$$
G_{i j}=\left\langle\delta w_{i} \delta w_{j}\right\rangle=\delta_{i j} u_{i}
$$

Let us introduce $\vec{\psi}$ for convenience as follows:

$$
\vec{\psi} \equiv \sqrt{2} \delta \vec{w}
$$

or

$$
\vec{\psi}=R \vec{\omega} \quad \text { and } \quad \vec{\omega}=R^{T} \vec{\psi}
$$

Because $e^{-\tilde{S}}$ should express a Gaussian distribution of momentum of the particles, and $\left\langle\delta w_{i} \delta w_{j}\right\rangle=0$ unless $i=j$,

$\tilde{S}=\sum_{i=1}^{3} \frac{\delta w_{i}^{2}}{2\left\langle\delta w_{i}^{2}\right\rangle}=\sum_{i=1}^{3} \frac{1}{2 u_{i}} \delta w_{i}^{2}=\frac{1}{4} \sum_{i=1}^{3} \frac{1}{u_{i}} \psi_{i}^{2}$.

On the other hand, from Eqs. (43) and (55),

$$
\tilde{S}=\frac{1}{4} \vec{\psi}^{T} R A R^{T} \vec{\psi}
$$

Then, $A$ is also diagonalized by $R$ as

$$
R A R^{T}=\left(\begin{array}{ccc}
\frac{1}{u_{1}} & 0 & 0 \\
0 & \frac{1}{u_{2}} & 0 \\
0 & 0 & \frac{1}{u_{3}}
\end{array}\right),
$$

which can also be derived from Eqs. (46), (50), and (51).

The diffusion rate of the normal momentum is expressed as

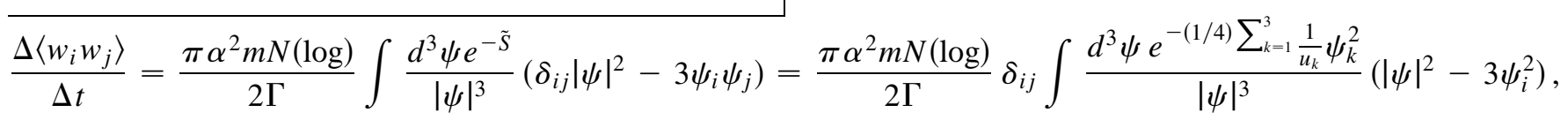

which is derived from Eq. (44) using

$$
\begin{gathered}
|\omega|=|\psi|, \\
\int d^{3} \omega=\int d^{3} \psi, \\
\sum_{k, l=1}^{3} R_{i, k} R_{j, l}\left\langle\delta p_{k} \delta p_{l}\right\rangle=\left\langle\delta w_{i} \delta w_{j}\right\rangle, \\
\sum_{k, l=1}^{3} R_{i, k} R_{j, l}\left(\delta_{k l}\right)=\left(\delta_{i j}\right), \\
\sum_{k, l=1}^{3} R_{i, k} R_{j, l}\left(\omega_{k} \omega_{l}\right)=\left(\psi_{i} \psi_{j}\right) .
\end{gathered}
$$

Replacing $\vec{p}$ by $\vec{w}$ and $\omega$ by $\psi$, Eq. (59) has the same form as Eq. (44) except the $\tilde{S}$, which has no cross term of $\psi$. Equation (59) can be expressed as

$$
\begin{aligned}
& \frac{\Delta\left\langle w_{1}^{2}\right\rangle}{\Delta t}=c_{I}\left[\left(g_{2}-g_{1}\right)+\left(g_{3}-g_{1}\right)\right], \\
& \frac{\Delta\left\langle w_{2}^{2}\right\rangle}{\Delta t}=c_{I}\left[\left(g_{1}-g_{2}\right)+\left(g_{3}-g_{2}\right)\right], \\
& \frac{\Delta\left\langle w_{3}^{2}\right\rangle}{\Delta t}=c_{I}\left[\left(g_{1}-g_{3}\right)+\left(g_{2}-g_{3}\right)\right],
\end{aligned}
$$

introducing

$$
g_{i} \equiv \frac{1}{4 \pi} \int \frac{d^{3} \psi e^{-(1 / 4) \sum_{k=1}^{3} \frac{1}{u_{k}}} \psi_{k}^{2}}{|\psi|^{3}} \psi_{i}^{2}
$$

and

$$
c_{I} \equiv \frac{2 \pi^{2} \alpha^{2} m N(\log )}{\Gamma}=\frac{r_{e}^{2} N(\log )}{4 \pi \gamma^{3} \epsilon_{1} \epsilon_{2} \epsilon_{3}},
$$

where $r_{e}$ is the classical electron radius, and $\epsilon_{1}, \epsilon_{2}$, and $\epsilon_{3}$ are the normal mode emittances in the laboratory frame. 
Since the quantity $g_{i}$ resembles the average squared momentum, it can be interpreted as temperature. $\Delta\left\langle w_{k}^{2}\right\rangle / \Delta t$ is heating in the direction $k$, and Eqs. (65) can be explained as "Heat transfer from one direction to another is proportional to the temperature difference between the two directions."

The integral of $d^{3} \psi$ can be transferred to the integral of one variable, which is more suitable for numerical calculations. Using

$$
\frac{1}{|\psi|^{3}}=\int_{0}^{\infty} \frac{d \lambda}{4 \sqrt{\pi}} \sqrt{\lambda} e^{-(\lambda / 4)|\psi|^{2}}
$$

as in BM, and replacing the variable as

$$
\begin{gathered}
\lambda=\frac{1}{u_{k}} \tan ^{2} s, \\
g_{1}=\int_{0}^{\pi / 2} \frac{2 u_{1} \sin ^{2} s \cos s d s}{\sqrt{\left(\sin ^{2} s+\frac{u_{1}}{u_{2}} \cos ^{2} s\right)\left(\sin ^{2} s+\frac{u_{1}}{u_{3}} \cos ^{2} s\right)}}, \\
g_{2}=\int_{0}^{\pi / 2} \frac{2 u_{2} \sin ^{2} s \cos s d s}{\sqrt{\left(\sin ^{2} s+\frac{u_{2}}{u_{1}} \cos ^{2} s\right)\left(\sin ^{2} s+\frac{u_{2}}{u_{3}} \cos ^{2} s\right)}}, \\
g_{3}=\int_{0}^{\pi / 2} \frac{2 u_{3} \sin ^{2} s \cos s d s}{\sqrt{\left(\sin ^{2} s+\frac{u_{3}}{u_{1}} \cos ^{2} s\right)\left(\sin ^{2} s+\frac{u_{3}}{u_{2}} \cos ^{2} s\right)}} .
\end{gathered}
$$

Finally, the diffusion rate of the momentum is calculated as

$$
\frac{\Delta\left\langle p_{i} p_{j}\right\rangle}{\Delta t}=\sum_{k=1}^{3} R_{i k} R_{j k} \frac{\Delta\left\langle w_{k}^{2}\right\rangle}{\Delta t} .
$$

\section{LOG FACTOR AND TAIL CUT}

The so-called Coulomb logarithm is an ambiguous part of the formula and is only roughly evaluated. However, as shown later, the result can be significantly different from other calculations, such as in Ref. [2].

This factor is from the integral of the scattering angle, $\theta$, in the center-of-mass frame of the two particles as follows:

$$
\begin{aligned}
(\log ) & =\frac{1}{2} \int_{\cos \theta_{\max }}^{\cos \theta_{\min }} d(\cos \theta) \frac{1}{1-\cos \theta} \\
& =\frac{1}{2} \log \frac{1-\cos \theta_{\max }}{1-\cos \theta_{\text {min }}},
\end{aligned}
$$

where $\theta_{\min }$ and $\theta_{\max }$ are the minimum and maximum angle, respectively. $\theta_{\min }=0$ makes the integral divergent, reflecting the long-distance Coulomb force.

Let us consider the impact parameter, $b$, of the scattering. For nonrelativistic Coulomb scattering, the impact parameter is expressed as a function of the scattering angle as

$$
b(\theta)=\frac{m \alpha}{|\vec{p}|^{2}} \cot \frac{\theta}{2} \approx \frac{2 m \alpha}{\theta|\vec{p}|^{2}},
$$

where $\vec{p}$ is the momentum of the particles in the center-ofmass frame, and the approximation is good for small $\theta$.
Let us define

$$
b_{\min } \equiv \frac{\sqrt{2} m \alpha}{\left\langle\delta \vec{p}^{2}\right\rangle \sqrt{1-\cos \theta_{\max }}} \approx b\left(\theta_{\max }\right) \approx \frac{2 m \alpha}{\theta_{\max }\left\langle\delta \vec{p}^{2}\right\rangle},
$$

$$
b_{\max } \equiv \frac{\sqrt{2} m \alpha}{\left\langle\delta \vec{p}^{2}\right\rangle \sqrt{1-\cos \theta_{\min }}} \approx b\left(\theta_{\min }\right) \approx \frac{2 m \alpha}{\theta_{\min }\left\langle\delta \vec{p}^{2}\right\rangle},
$$

so that we may set $b_{\min }$ and $b_{\max }$ instead of $\theta_{\max }$ and $\theta_{\min }$, and the log factor is expressed as

$$
(\log )=\log \frac{b_{\max }}{b_{\min }} \approx \log \frac{\theta_{\max }}{\theta_{\min }} .
$$

The approximation is good if

$$
b_{\max }>b_{\min } \gg b(\pi / 2)=\frac{m \alpha}{\left\langle\delta \vec{p}^{2}\right\rangle} .
$$

$\vec{p}^{2}$ was replaced by the square of a typical momentum, $\left\langle\delta \vec{p}^{2}\right\rangle$.

We did not include following process in the calculation: (i) scattering with impact parameter larger than the beam size, (ii) scattering with impact parameter larger than the mean distance between two closest particles, (iii) rare scattering with small impact parameter, whose rate is smaller than the radiation damping rate.

The first two criteria mean

$$
b_{\max }=\min \left(\langle\varrho\rangle^{-1 / 3}, \sqrt{v_{1}}, \sqrt{v_{2}}, \sqrt{v_{3}}\right),
$$

where $\langle\varrho\rangle$ is the average of particle density and $v_{1}, v_{2}, v_{3}$ are the eigenvalues of the beam spatial matrix $\left\langle x_{i} x_{j}\right\rangle$. Reference [2] uses $(\log )=20$ instead of stating what $b_{\max }$ should be. Reference [1] takes $b_{\max }=\langle\varrho\rangle^{-1 / 3} / 2$ and Ref. [3] takes $b_{\max }=\langle\varrho\rangle^{-1 / 3}$. We take the beam size of the minimum direction if it is smaller than the mean distance between two closest particles. Other discussions are found. For example, Ref. [12] suggests taking the beam radius instead of the mean distance of particles. Note that the choice of $b_{\max }$ is still ambiguous.

The last criterion is interpreted as so-called "tail cut," which was proposed by Raubenheimer [13,14], but was not considered in Refs. [1-3]. Actual beam distribution may not be Gaussian. On the other hand, in our calculation the distribution is assumed to be Gaussian, and only the core part of the beam should be considered. Therefore large angle collisions whose rate is less than the radiation damping rate and which make a beam tail should be eliminated. This last criterion can be expressed as

$$
\sigma_{\theta>\theta_{\max }} v\langle\varrho\rangle=\frac{1}{\tau_{\text {rad }}},
$$

where $v=\sqrt{u_{1}+u_{2}+u_{3}} / m$ is the typical velocity of the particles and $\tau_{\text {rad }}=\max \left(\tau_{1}, \tau_{2}, \tau_{1}\right)$ is the longest radiation damping time in the rest frame of the beam. Note that the damping time in the rest frame is shorter than that in the laboratory frame by a factor of $\gamma, \tau_{\text {rad }}=\tau_{\text {rad,lab }} / \gamma$. 

is

A cross section with a scattering angle larger than $\theta_{\max }$

$$
\sigma_{\theta>\theta_{\max }}=2 \pi\left(\frac{m \alpha}{\left\langle\delta \vec{p}^{2}\right\rangle}\right)^{2} h\left(\theta_{\max }\right),
$$

where $h\left(\theta_{\max }\right)$ is defined as

$$
h\left(\theta_{\max }\right)=\int_{\theta_{\max }}^{\pi / 2} d(\cos \theta)\left(\frac{4}{\sin ^{4} \theta}-\frac{3}{\sin ^{2} \theta}\right) .
$$

If $\theta_{\max }$ is small,

$$
h\left(\theta_{\max }\right) \approx \frac{1}{\left(1-\cos \theta_{\max }\right)},
$$

and

$$
b_{\min } \approx \sqrt{\frac{1}{\pi\langle\varrho\rangle v \max \left(\tau_{1}, \tau_{2}, \tau_{3}\right)}}
$$

If $\theta_{\max }$ is not very small, we can use Eqs. (74) and (81) and

$$
h\left(\theta_{\max }\right)=\frac{1}{\left.2 \pi\left(\frac{m \alpha}{\langle\vec{\delta} p\rangle}\right\rangle\right)^{2} v\langle\varrho\rangle \max \left(\tau_{1}, \tau_{2}, \tau_{3}\right)} .
$$

Note that, in such a case, $b_{\min }$ can no longer be interpreted as the minimum impact parameter.

In usual accelerators, $b_{\max }$ is larger than $b_{\text {min }}$ by many orders of magnitude, and the result depends only on its logarithm. For that reason, the approximation of Eq. (83) is not bad unless the right-hand side is extremely small. Then it is a good approximation to set

$$
b_{\min } \approx b_{\min }^{\prime} \equiv \max \left(\frac{\sqrt{2} m \alpha}{\left\langle\vec{\delta}^{2} p\right\rangle}, \sqrt{\frac{1}{\pi \bar{\varrho} v \max \left(\tau_{1}, \tau_{2}, \tau_{3}\right)}}\right) .
$$

$b_{\min }^{\prime}$ and $b_{\min }$ are numerically compared in Fig. 1, where both are divided by $\sqrt{2} b(\pi / 2)$. The relation is close to a straight line with unit slope showing the validity of the approximation.

As an example, let us roughly estimate the log factor for the Accelerator Test Facility (ATF) damping ring at KEK.

$b_{\max }$ is evaluated from

$$
\langle\varrho\rangle=\frac{1}{N} \int d^{3} x \varrho^{2}(x)=\frac{N}{2^{3} \pi^{3 / 2} \sqrt{v_{1} v_{2} v_{3}}},
$$

with

$$
\sqrt{v_{1,2}} \approx \sigma_{x, y}=\sqrt{\epsilon_{x, y} \beta_{x, y}}
$$

and

$$
\sqrt{v_{3}} \approx \sigma_{z}=\gamma \sigma_{z, \mathrm{lab}}
$$

where $\varrho(x)$ is the particle density, $\sigma_{x, y, z}$ the horizontal, vertical, or longitudinal beam size, $\epsilon_{x, y}$ the emittance, $\beta_{x, y}$ the beta function, and $\sigma_{z}$, lab the longitudinal beam size in the laboratory frame.

$b_{\text {min }}$ is evaluated from

$$
\left\langle\vec{\delta} p^{2}\right\rangle \approx m^{2} v^{2}
$$

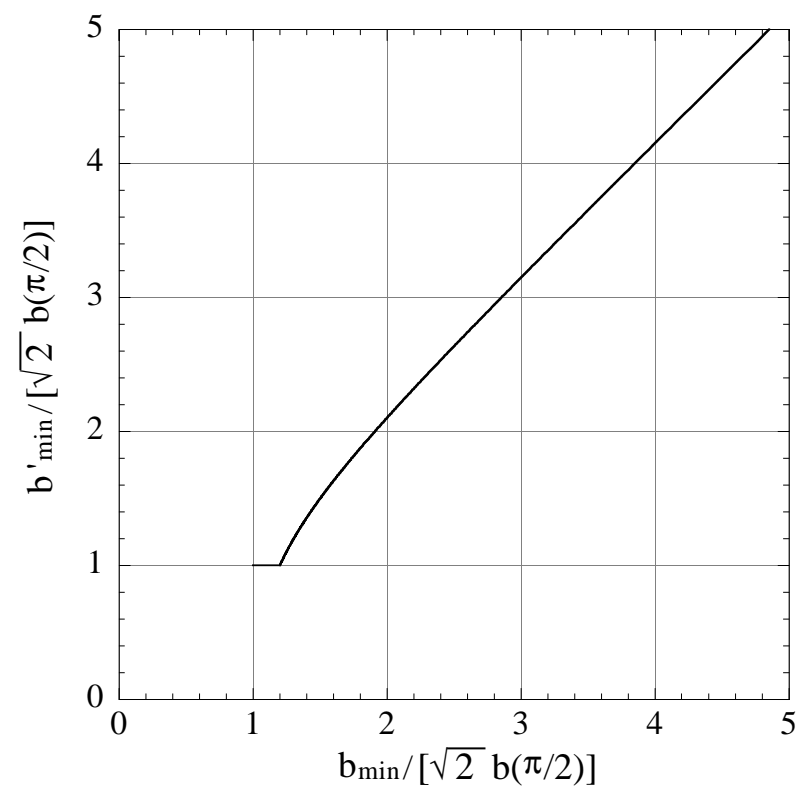

FIG. 1. $\quad b_{\min }^{\prime} / b(\pi / 2)$ (the solid line) versus $b_{\min } / b(\pi / 2)$ from a numerical calculation.

$$
v \approx c \gamma \sigma_{x^{\prime}, \mathrm{lab}} \approx c \gamma \sqrt{\frac{\epsilon_{x}}{\beta_{x}}},
$$

where $\sigma_{x^{\prime} \text {,lab }}$ is the rms of the horizontal angle in the laboratory frame.

Using the parameters in Table I, we get

$$
\begin{gathered}
b_{\max }=\langle\varrho\rangle^{-1 / 3} \approx 3 \times 10^{-6} \mathrm{~m}, \\
b(\pi / 2) \approx 1 \times 10^{-12} \mathrm{~m}
\end{gathered}
$$

and

$$
b_{\min }=2 \times 10^{-10} \mathrm{~m} .
$$

The $\log$ factor is

$$
(\log )=\log \frac{b_{\max }}{b_{\min }} \approx 10 .
$$

$b_{\max }$ is larger than $b_{\text {min }}$ by a factor of $10^{4}$, and our approximation is justified in the case of the ATF damping ring.

This result is significantly different from a calculation without the tail cut, $b_{\min } \approx \sqrt{2} m \alpha /\left\langle\vec{\delta} p^{2}\right\rangle$ and $(\log ) \approx 15$. It is also different from Ref. [2], in which $(\log )=20$.

TABLE I. Parameters of the ATF damping ring.

\begin{tabular}{lc}
\hline \hline Number of electrons per bunch $N$ & $\approx 1 \times 10^{10}$ \\
Energy factor $\gamma$ & $2.6 \times 10^{3}$ \\
Horizontal emittance $\epsilon_{x}$ & $\sim 1 \times 10^{-9}$ \\
Vertical emittance $\epsilon_{y}$ & $\sim 1 \times 10^{-11}$ \\
Beta functions $\beta_{x}$ and $\beta_{y}$ & $\sim 3 \mathrm{~m}$ \\
Bunch length $\sigma_{z, \text { lab }}$ & $6 \mathrm{~mm}$ \\
Damping times $\tau_{x, \text { lab }}, \tau_{y, \text { lab }}, \tau_{z, \text { lab }}$ & $17,27,20 \mathrm{~ms}$ \\
\hline \hline
\end{tabular}




\section{CALCULATION OF EMITTANCE}

As described in the previous sections, once we know the local momentum matrix $F_{i j}=\left\langle\delta p_{i} \delta p_{j}\right\rangle$ at a certain position of an accelerator, we can find an orthogonal matrix to diagonalize it and calculate $\Delta\left\langle\delta w_{i}^{2}\right\rangle / \Delta t$ and then $\Delta\left\langle\delta p_{i} \delta p_{j}\right\rangle / \Delta t$, the diffusion rate of the momentum. Dividing an accelerator into short pieces, the change of the momentum matrix at each position can be calculated. Note that the rate expressed in the beam rest frame is different from the rate expressed in the laboratory frame by the energy factor $\gamma$, due to a relativistic time expansion.

A method for calculating the equilibrium emittance of an electron beam in a storage ring is described in Ref. [15]. The quantum excitation due to the synchrotron radiation is included as a momentum diffusion process as follows:

$$
R(s)=M\left(s, s_{0}\right) R\left(s_{0}\right) M^{T}\left(s, s_{0}\right)+\bar{B}\left(s, s_{0}\right),
$$

where $R$ is the $6 \times 6$ beam-envelope matrix, $M$ the transfer matrix, and $\bar{B}$ the integrated diffusion matrix,

$$
\bar{B}\left(s, s_{0}\right)=\int_{s_{0}}^{s} M\left(s, s_{1}\right) B\left(s_{1}\right) M^{T}\left(s, s_{1}\right) d s_{1},
$$

where $B$ is called the diffusion matrix.

The effect of the intrabeam scattering can also be included as a momentum diffusion in the similar way as the synchrotron radiation. The diffusion matrix for the intrabeam scattering can be evaluated from the matrix $\Delta\left\langle\delta p_{i} \delta p_{j}\right\rangle / \Delta t$. The only difference is that the intrabeam scattering depends on the beam size, whereas the synchrotron radiation does not. Because the intrabeam scattering changes the beam size, some iterations are necessary to obtain a self-consistent result.

This method is used in a computer program SAD [7] for calculating the equilibrium beam emittance in an electron storage ring.

\section{ACKNOWLEDGMENTS}

This work was performed closely related to the ATF experiments, and the authors thank all members of the ATF collaboration, especially J. Urakawa and H. Hayano for leading the ATF project. They also thank K. Bane for useful discussions and suggestions about theories and comparisons with experimental data.

[1] A. Piwinski, in Proceedings of the 9th International Conference on High Energy Accelerators, Stanford, CA, 1974 (National Technical Information Service, Springfield, VA, 1975), p. 405.

[2] J. Bjorken and S. K. Mtingwa, Part. Accel. 13, 115 (1983).

[3] J.L. Duff, in Proceedings of the CERN Accelerator School: Second Advanced Accelerator Physics Course, Berlin, 1987 (CERN, Geneva, 1989), p. 573.

[4] K. Kubo, in Proceedings of the 18th International Conference on High Energy Accelerators, Tsukuba, 2001 (Report No. ID TU-02, 2001).

[5] K. Bane et al., in Proceedings of the 2001 Particle Accelerator Conference, Chicago (unpublished).

[6] C. Steier et al., in Proceedings of the 2001 Particle Accelerator Conference, Chicago (unpublished).

[7] A computer code, SAD (strategic accelerator design), was developed at KEK. For example, see K Hirata, in Proceedings of the 2nd International Committee for Future Accelerator Beam Dynamics Workshop, Lugano, 1988, edited by E. Keil and J. Hagel (CERN Report No. 88-04, 1988).

[8] For example, James D. Bjorken and Sideny D. Drell, Relativistic Quantum Mechanics (McGraw-Hill, New York, 1964).

[9] T. Toyomasu, Part. Accel. 40, 161 (1993) (relativistic effects are considered in this reference).

[10] From some equations in Ref. [2], this factor appears to be $q_{i} \xi_{j}+q_{j} \xi_{i}+q_{i} \Delta_{j}+q_{j} \Delta_{i}+2 q_{i} q_{j}$. But this difference has no significance because the odd terms of $\xi$ vanish after the integration.

[11] Here we use $\sigma_{\delta}$ instead of $\sigma_{\eta}=\sqrt{2} \sigma_{\delta}$, which is used in Ref. [2]. In this reference the equation $\Gamma_{\text {unbunch }}=$ $(2 \pi)^{3} \beta^{3} \gamma^{3} \epsilon_{x} \epsilon_{z} \sigma_{\eta} \sigma_{s}$ appears, where $\epsilon_{x}$ and $\epsilon_{z}$ are the horizontal and vertical emittances and $\sigma_{s}$ is the bunch length. It should be $\Gamma_{\text {unbunch }}=(2 \pi)^{3} \beta^{3} \gamma^{3} \epsilon_{x} \epsilon_{z} \sigma_{\delta} \sigma_{s}$. The difference is by a factor of $\sqrt{2}$.

[12] R. T. Farouki and E. E. Salpeter, Astrophys. J. 427, 676 (1994).

[13] T. Raubenheimer, SLAC Report No. SLAC-387, 1991, Sec. 2.3.1.

[14] T. Raubenheimer, Part. Accel. 45, 111 (1994).

[15] K. Ohmi, K. Hirata, and K. Oide, Phys. Rev. E 49, 751 (1994). 